\title{
THE ABSORPTION, EXCRETION, AND DESTRUCTION OF ORALLY ADMINISTERED PENICILLIN ${ }^{1}$
}

\author{
By WALSH MCDERMOTT, PAUL A: BUNN, MARIA BENOIT, REBECKAH \\ DuBOIS, AND MILDRED E. REYNOLDS
}

(From the Department of Medicine, Cornell University Medical College and The New York Hospital, New York City)

(Received for publication October 29, 1945)

\section{INTRODUCTION}

In the original investigations of the pharmacology of penicillin by $\mathrm{H}$. Florey and colleagues (1), it was demonstrated that the material was absorbed from the alimentary tract following. oral or intraduodenal administration. These studies included observations on two normal subjects, one patient with an infection, and a series of experimental animals. As it was known from experience in the production of penicillin that inactivation of the material occurs rapidly at $\mathrm{pH} 2$, these investigators (2) attempted to protect orally administered penicillin from destruction by the acid of the stomach by the use of "enteric" coated capsules. Dissolution of the capsules did not occur uniformly, so that absorption was irregular. As the available supply of penicillin was so limited at that time, further study of oral administration was not pursued.

A similar investigation was conducted by Rammelkamp and Keefer (3), who detected small amounts of penicillin in the blood and urine of three subjects who ingested 10,000 to 20,000 units of the material in aqueous solution. The administration of alkali with the penicillin appeared to increase the absorption of the latter. When penicillin was introduced into the rectum, only minute amounts were absorbed and excreted in the urine. Further studies of the problem of oral administration were reported in subsequent publications by Rammelkamp and Helm (4). The incubation of penicillin in broth adjusted to various levels of $\mathrm{pH}$ by the addition of one-tenth normal hydrochloric acid was followed by the rapid destruction of the penicillin in the lower ranges of $\mathrm{pH}$. Incubation with succus entericus caused no inactiva-

1 The work described in this paper was done under a contract, recommended by the Committee on Medical Research, between the Office of Scientific Research and Development and Cornell University Medical College. tion of penicillin. As absorption of ingested penicillin was irregular, and as the use of the route was wasteful of the material, the oral administration of penicillin was not considered to be feasible at that time.

In addition to the above reports, Powell and Jamieson, and Libby $(5,6)$ had noted that mice could be protected against lethal doses of pneumococci and other organisms by the administration of penicillin in the diet. Free and his associates (7) had also found that 8 to 33 per cent of a dose of 100,000 units of penicillin could be detected in the urine during the six hours immediately following ingestion. Although these initial studies of the oral administration of penicillin were by no means discouraging, an extended investigation of the problem had to await the production of a larger supply of the material.

Almost simultaneously, there appeared, early in 1945 , publications from five laboratories. $(6,8,9$, $10,11)$ in which it was reported that the oral administration of penicillin was practicable.

In four of these investigations, attempts had been made to protect the penicillin from destruction by the acid of the stomach by the use of an oil suspension (Libby), admixture with egg white (Little and Lumb), or combination with a tricitrate buffer (György, Charney, and their respective associates). By all of these methods, concentrations of penicillin in the blood or urine comparable to those usually observed following intramuscular administration were attained, albeit with the use of considerably more penicillin. In the other publication (11), which was by the authors of the present communication, it was reported that when different methods of oral administration were compared in the same fasting subjects there was no demonstrable difference in the concentrations attained in blood and urine following the oral administration of penicillin in 
an oil suspension, in an oil suspension mixed with beeswax, after the administration of an antacid (magnesium trisilicate), or in aqueous solution. (György et al, and Charney et al. also noted that in different fasting subjects the penicillin tricitrate preparation did not seem to be more efficient than the aqueous solution.) With any of the four methods of oral administration studied by us, approximately five times as much penicillin was required to produce a given concentration in the blood as by the use of the intramuscular route.

In an extension of these studies, essentially similar results were obtained following the administration of penicillin with a number of other vehicles which included: penicillin-sodium-tricitrate tablets; penicillin adsorbed on aluminum hydroxide; penicillin-mucin; penicillin-lecithin; penicillin-oil-shellac; and penicillin-oil-beeswax-shellac. With any of these vehicles, it was necessary to use large quantities (approximately five times the intramuscular dose) to promote the absorption of appreciable amounts of penicillin.

It appeared, therefore, that before rational attempts could be made to increase the absorption of ingested penicillin it was essential to obtain more information about the mechanisms of the absorption, excretion, and destruction of the orally administered material. Accordingly, the present investigation was undertaken.

\section{MATERIAL AND METHODS}

\section{Penicillin:}

Unless otherwise stated, the partially purified sodium and calcium salts of penicillin were used.

\section{Bioassay of penicillin:}

Penicillin determinations were performed by the serial dilution technique of Rammelkamp (12) in the first part of the study, and more recently by the Kirby-Rantz (13) modification of this. technique. The organism used in both tests was the Group A beta-hemolytic streptococcus C203Mv. Crystalline sodium penicillin G 2 (1650 units per $\mathrm{mgm}$.) was used as the penicillin standard. Under the conditions existing throughout the period of the investigation, the maximum sensitivity in the detection of penicillin in an unknown specimen was 0.078 unit per $\mathrm{ml}$. for the Rammelkamp method and 0.04 unit per $\mathrm{ml}$. for the Kirby-Rantz modification. The blood penicillin determinations were all made on specimens of serum. Specimens of urine and gastric juice were collected without a preservative and immediately subjected to Seitz filtration.

2 Obtained through the courtesy of the late Dr. George Harrop of Squibb Institute.
In the experiments in which penicillin was incubated at $37^{\circ} \mathrm{C}$. with specimens of gastric juice adjusted to various hydrogen ion concentrations, the specimens were rendered neutral or alkaline at the end of the experimental period and before the penicillin determinations were made.

Determinations of penicillin in feces were made by emulsifying an aliquot of stool in five times the volume of distilled water. The emulsion was then centrifuged at 1500 revolutions per minute for 20 to 30 minutes. The supernatant fluid was filtered through Whatman filter paper which had been previously covered with approximately 5.0 grams of diatomaceous earth. (Penicillin is not adsorbed from aqueous solution at $\mathrm{pH} 6$ to 7 by this agent.) The resulting filtrate was then passed through a Seitz filter and assayed in the same manner as the other specimens. In more recent experiments, the preliminary filtration through diatomaceous earth was omitted.

All specimens from any source which were not assayed on the day on which they were obtained were maintained in a sterile condition at a temperature of $-28^{\circ} \mathrm{C}$. until the penicillin determinations were made. All the determinations of $\mathrm{pH}$ were made with the Beckman meter.

\section{Animals:}

In the cat experiments the animals were anesthetized by the intraperitoneal administration of Dial $(50 \mathrm{mgm}$. per $\mathrm{kgm}$.). The stomach was tied and surgically severed through the pylorus. The penicillin was introduced into the stomach through a stomach tube or by direct puncture with a needle. The intestinal segments were usually isolated from the rest of the gut by ties placed proximal and distal to the point where the penicillin was introduced by needle. In a few experiments the proximal tie was omitted.

In the dog experiment, ether was used as an anesthetic. The oral dose of penicillin was administered in a capsule with a small piece of meat, and the intravenous injection was made directly into the portal vein under direct exposure.

\section{Subjects:}

The human subjects were all studied in the morning after an overnight fast. Most of the subjects were healthy adults with presumably normal acid secretion, but in some experiments hospital patients with no demonstrable renal or gastrointestinal disease were studied. One subject (Tom) has a permanent gastric fistula and has served as the subject of an extensive investigation of gastric function.

\section{EXPERIMENTAL RESULTS}

In 9 cats, determinations of the blood penicillin concentrations were made at constant intervals after introduction of the aqueous solution of penicillin into previously isolated divisions of the gastrointestinal tract. The dose of penicillin 

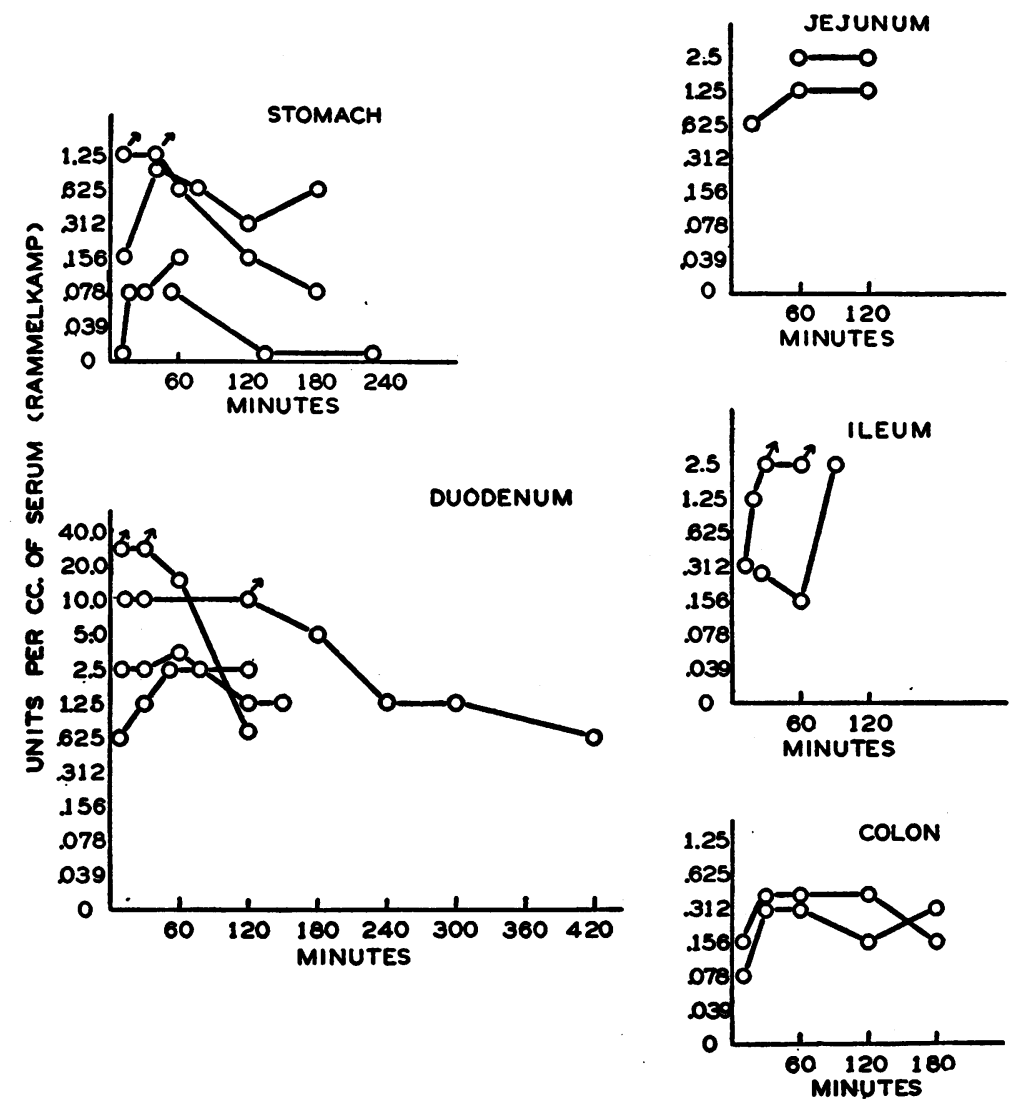

Fig. 1. Absorption of Penicillin from Isolated Segments of the Gastrointestinal Tract of Cats

was 15,000 units or approximately 5,000 units per $\mathrm{kgm}$. (the equivalent of a 300,000 unit dose in a $60 \mathrm{kgm}$. adult). As may be seen in Figure 1, the greatest absorption occurred when the penicillin was administered into an isolated segment of duodenum. The maximum concentrations attained ranged from 2.5 to more than 20.0 units per $\mathrm{ml}$. of serum. When the penicillin was confined in a segment of jejunum or ileum, the absorption was almost as great as from the duodenum. Absorption from the large bowel occurred but was distinctly less than from the small intestine. The absorption from the stomach was also less than from the small intestine and varied considerably in the individual experiments.

In contrast to the conditions which exist following the oral administration of penicillin in man, the penicillin in these experiments was confined to a small section of intestine throughout the experimental period. The persistence of the penicillin in the blood, once the maximum concentra- tion had been attained, was significantly longer than has been observed in man after the attainment of similar penicillin concentrations following administration by the intramuscular or the oral route. This would indicate either that absorption was continuing throughout the experimental period ( 1 to 7 hours) or that penicillin is eliminated less rapidly from the blood of the cat than occurs in man. Against the latter possibility is the fact that Florey et al. (2) observed in cats that the disappearance of penicillin from the blood and its excretion in the urine proceeded in a fashion comparable to what had been observed in man.

Aside from the question of the duration of penicillin activity in the blood, it is probable that the extent of absorption of penicillin which occurred from the various sections of the gastrointestinal tract of the cat is similar to the absorption from the same areas in man, i.e., greatest absorption from the duodenum, least from the large intestine and a variable but small amount from the stomach. 
Injection of penicillin into portal vein:

The possibility that the liver might destroy an appreciable amount of orally administered penicillin after absorption into the portal circulation was investigated. On 3 successive days the same dose of penicillin, 50,000 units (approximately 3,000 units per $\mathrm{kgm}$.), was administered orally, intramuscularly, and by direct injection into the portal vein of a dog.

The maximum concentrations of penicillin attained in the systemic venous blood were: 20 units per $\mathrm{ml}$. after injection into the portal vein, 10 units per ml. after intramuscular injection, and 0.312 units per $\mathrm{ml}$. following oral administration.

In similar experiments in 2 cats, the penicillin concentrations attained in the systemic venous blood following introduction of the material directly into the portal vein were of the same order of magnitude as were attained following injection into a systemic vein.

Thus in these three experiments there was no indication that the liver prevented the transfer of penicillin from the portal to the systemic circulation.

Destruction of penicillin in the stomach:

A series of experiments were performed to investigate the validity of the widely held assump- tion that the relative inefficiency of the oral route of administration is due to destruction of penicillin by the acid of the stomach.

\section{Effect of the $p H$ of gastric juice on the destruction of penicillin in vitro:}

In preliminary experiments in vitro, determinations were made of the rate of inactivation of crystalline sodium penicillin $G$ by incubation at $37^{\circ} \mathrm{C}$. with gastric juice which had been adjusted to various levels of $\mathrm{pH}$. The results were in agreement with the previous report of Rammelkamp and Helm (4) on the destruction of partially purified penicillin in gastric juice and in broth.

The destruction of the crystalline penicillin was rapid at a $\mathrm{pH}$ of 2.0 , the process being completed within less than 1 hour. At levels of $\mathrm{pH}$ between 3.0 and 4.0, considerable penicillin was destroyed during the first 15 minutes, with less rapid destruction during the remainder of the ex- . perimental period ( 4 hours). The inactivation of the material kept at or above $\mathrm{pH} 4.0$ proceeded less rapidly and was not complete until the fourth hour. At $\mathrm{pH} 7.2$ to 7.5, there was no inactivation of penicillin during the period of observation (4 hours). Moreover, no inactivation was demonstrable in experiments in which penicillin was

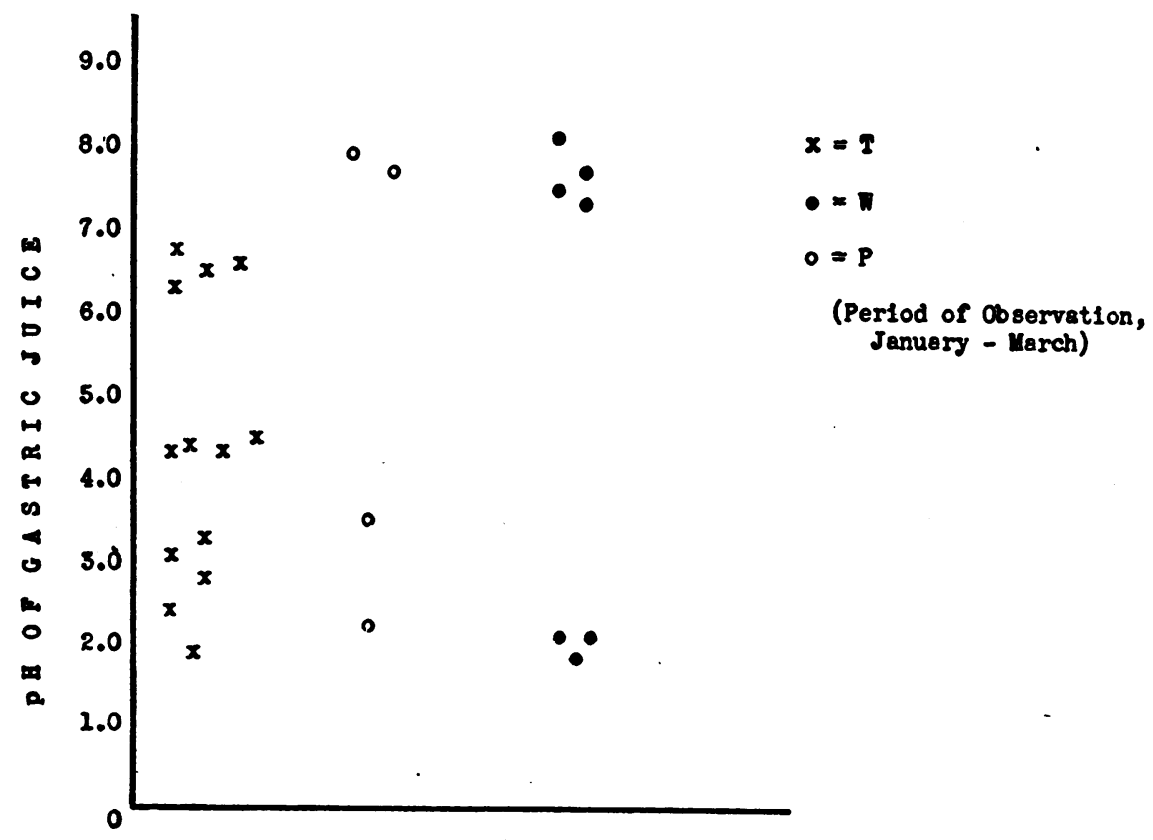

Fig. 2. The pH of the Fasting Gastric Content on Different Days in Three Individuals with Normal Acto Secretion 
incubated at $\mathrm{pHs} 7.8$ and 8.1 with specimens of gastric juice obtained from: $(a)$ an individual with normal acid secretion; and (b) a patient with pernicious anemia. It appears, therefore, that gastric juice per se does not inactivate penicillin and that the rate of destruction by the acidity of the gastric juice is appreciably slowed as the $\mathrm{pH}$ is raised, until at $\mathrm{pH} 4.0$ approximately 4 hours are required for the process to be completed.

The pH of the fasting gastric content of normal subjects:

In 3 subjects with normal acid secretion, determinations of the $\mathrm{pH}$ of the fasting gastric content were made repeatedly over a period of 6 months (Figure 2). In each subject there was a marked variation in the degree of acidity which was present on different days. The range in subject $\mathrm{I}(\mathrm{T})$ was from $\mathrm{pH} 1.95$ to $\mathrm{pH} 6.95$, in subject II (W) from $\mathrm{pH} 1.95$ to $\mathrm{pH} 8.1$, and in subject III (P) from $\mathrm{pH} 2.2$ to $\mathrm{pH}$ 7.9. A striking feature is the fact that in 14 of the total of 24 determinations, the $\mathrm{pH}$ of the fasting gastric content was above the level at which the destruction of penicillin is rapid in vitro $(\mathrm{pH} 4.0$ or higher).

Single determinations were made of the $\mathrm{pH}$ of the fasting gastric juice of 23 adults. All of these subjects secreted free hydrochloric acid after a subcutaneous injection of $0.33 \mathrm{mgm}$. of histamine phosphate. As they were all ambulatory patients in whom a gastric analysis had been ordered as part of a diagnostic investigation, it must be pre-
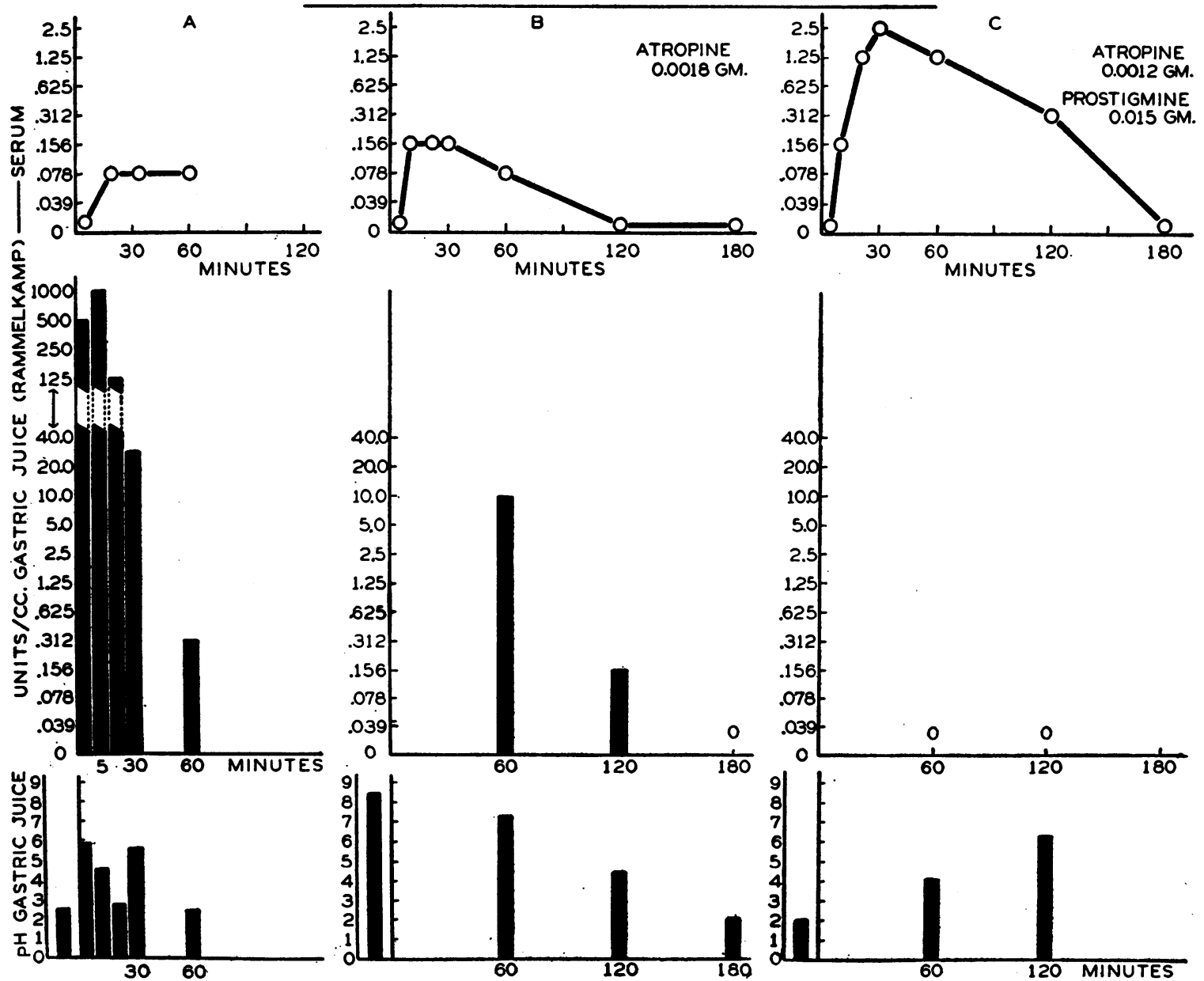

Fig. 3. Relation of the Penicillin Concentrations of the Blood After Ingestion of 300,000 Units, to the Penicillin Concentrations and the pH of the Gastric Content 

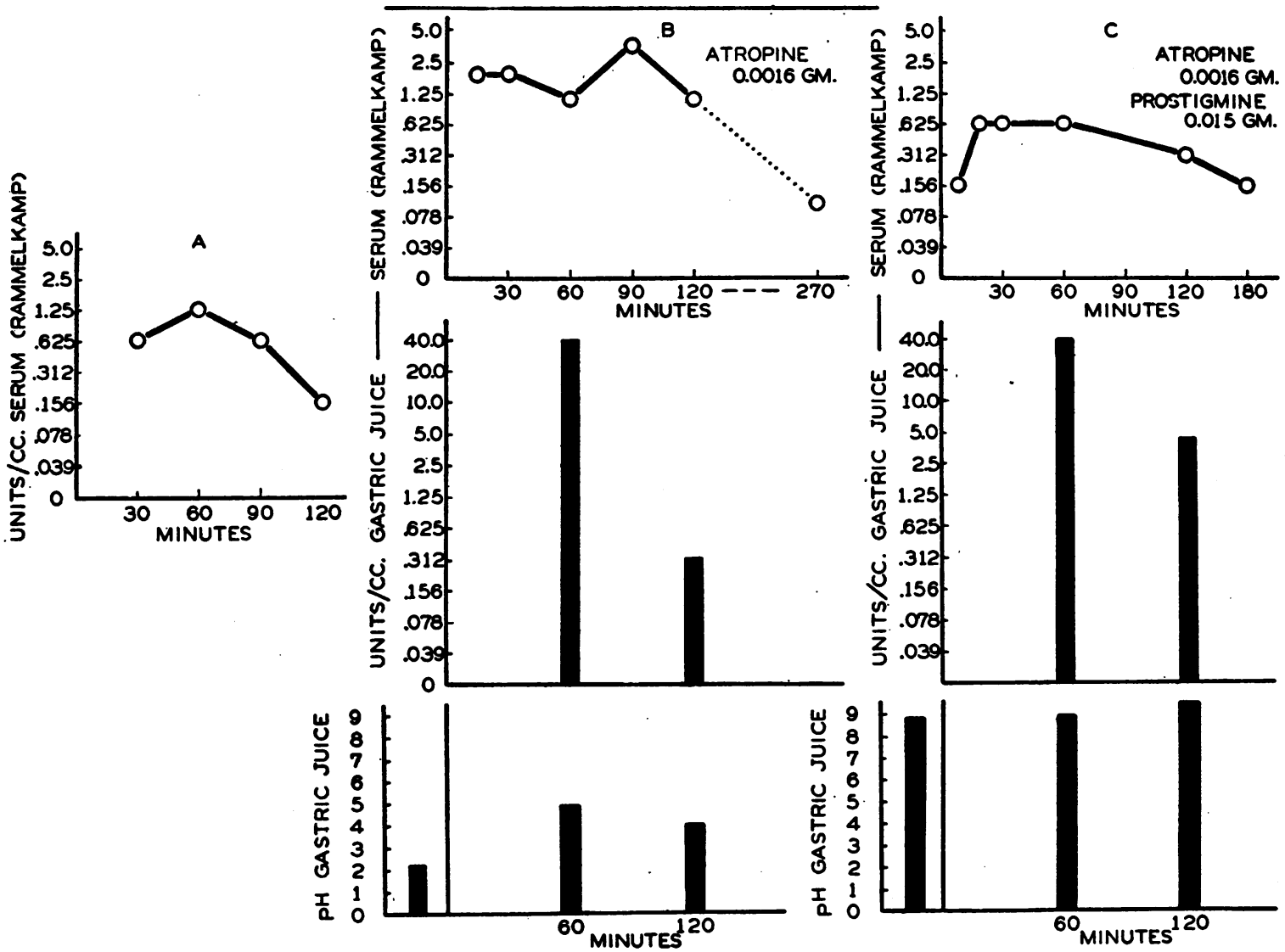

Fig. 4. Relation of the Penicillin Concentrations of the Blood After Ingestion of 300,000 Units, to the Penicillin Concentrations and the pH of the Gastric Content

sumed that the majority had symptoms referable to the gastrointestinal tract. A review of the records of these patients disclosed that no gastrointestinal disease was eventually demonstrable in any of the group of 23 . The $\mathrm{pH}$ of the gastric juice of 11 patients was below 3.0, in an additional 5 patients it was between 3.0 and 4.5 , and in 7 of the group it was $\mathbf{5 . 5}$ or higher.

Thus the $\mathrm{pH}$ of fasting gastric juice obtained from 3 individuals who were studied repeated$1 y$, and from other individuals in whom single determinations were made was frequently above the level $(\mathrm{pH} 4.0)$ at which the destruction of penicillin is rapid in vitro.

Inactivation of penicillin by acid in vivo:

In. the following experiments an attempt was made: (a) to study the inactivation of penicillin in the stomach; and $(b)$ to see if such inactivation could be related to the extent of absorption as de- termined by the height and duration of the penicillin levels attained in the blood. Two healthy males were the experimental subjects. A tube was passed into the stomach of each subject after a 15 hour fast. A sample of gastric content was withdrawn, and 300,000 units of penicillin dissolved in $30.0 \mathrm{ml}$. of water were administered through the tube. Specimens of gastric content and venous blood were obtained at intervals and were assayed for penicillin. In all but one experiment, the tube was removed after the individual aspirations. As it was impossible to measure the volume of fluid present in the stomach throughout the experiment, the concentration of the penicillin is expressed as units per $\mathrm{ml}$. of gastric juice. The results are presented in Figures 3 and 4.

It was impossible to determine how much of the change in penicillin concentration was a result of inactivation and how much was due to the con- 
tinuous dilution by the gastric secretions and the periodic emptying of the stomach. The highest concentration which was determined was 1,000 units per ml. 5 minutes after ingestion (Figure 3a). This value would indicate that the penicillin had been diluted with $270 \mathrm{ml}$. of gastric juice, a supposition which is not unreasonable. Aside from the question of inactivation, it is probable that a large part of the penicillin leaves the stomach within the first hour after ingestion.

Despite these several variables, it is noteworthy that appreciable concentrations of penicillin $(0.156$ unit per ml. to 100 units per ml.) were present in the stomach at 60 and 120 minutes after administration in 4 of the 5 experiments (Figures 3 and 4). In the experiment in which no penicillin was demonstrable in the gastric content, an attempt had been made to increase the emptying of the stomach by the administration of atropine and prostigmine, according to the technique of Wolf (15).

The levels of the $\mathrm{pH}$ of the gastric content at the time of the introduction of the penicillin were 2.0 , $2.18,2.2,7.9$, and 8.1 respectively. In the one experiment in which determinations were made soon after the introduction of the penicillin solution ( $\mathrm{pH}$ 6.5), a considerable rise in the $\mathrm{pH}$ occurred. As may be seen in Figure 3a, the initial $\mathrm{pH}$ of 2.18 was raised to 5.6 within one minute of the ingestion of the penicillin. During the succeeding hour, the $\mathrm{pH}$ of the gastric content fluctuated between 2.2 and 5.3.

No correlation between the degree of acidity and the concentration of penicillin in the gastric juice can be made because of the rapid fluctuations of $\mathrm{pH}$ and the fact that in some of the experiments an attempt was made to delay gastric emptying by the use of atropine. However, a comparison of the experiments presented in Figures $3 \mathrm{~b}$ and $4 \mathrm{~b}$ is of interest. In the former, the $\mathrm{pH}$ of the gastric content was above 8.0 when the penicillin was ingested and was at 7.0 at the end of one hour at which time the penicillin concentration in the stomach was 10 units per $\mathrm{ml}$. In contrast, in the experiment of Figure $4 \mathrm{~b}$, the penicillin concentration in the stomach was 40.0 units per $\mathrm{ml}$. one hour after ingestion, despite the fact that the $\mathrm{pH}$ levels of the gastric content were 2.0 and 4.8 respectively at the beginning and the end of the hour.
There was also no exact correlation between the maximum concentration of penicillin in the blood and the $\mathrm{pH}$ of the gastric juice. In the two experiments (Figures $3 c$ and $4 b$ ) in which the highest concentrations of penicillin were attained in the blood, the levels of the $\mathrm{pH}$ of the gastric juice at the beginning and the end of the first 60 minutes were 2.0 and 4.0, and 2.2 and 4.2, respectively. The poorest absorption was noted in the experiment (Figure 3a) in which the $\mathrm{pH}$ of the gastric juice was at or near 2.0 throughout a large part of the first 60 -minute period of observation. The amount of absorption in this experiment may have been modified by the presence of the tube in the stomach during the first half hour. It is of interest that in several experiments (Figures $3 \mathrm{~b}$ and $4 \mathrm{c}$ ), the maximum concentration in the blood was attained 20 minutes after ingestion and was falling during the second and third hours despite the continued presence of appreciable amounts of penicillin in the stomach.

Attempts to promote absorption by increasing the speed of gastric emptying by the administration of atropine and prostigmine led to results which were inconclusive (Figures $3 c$ and $4 c$ ).

Although, because of the many variables, it is impossible to evaluate precisely the results of these observations on the behavior of penicillin in the stomach, the data tend to support those obtained from the in vitro experiments. Even when all of these variables (fluctuations of $\mathrm{pH}$, variability of gastric emptying, effects of atropine and prostigmine, volume of gastric secretion) are taken into consideration, there are two observations which are worthy of note. First, appreciable amounts of penicillin were usually present in the gastric content one hour after ingestion of a 300,000 unit dose. Second, the absorption of a 300,000 unit ingested dose in subject II (Figure $4 c)$ at a time when his gastric content was consistently alkaline, was no greater (as judged by blood penicillin concentrations) than has frequently been observed in individuals with presumably normal acid secretion, and was actually less than when he ingested the same dose into gastric juice of $\mathrm{pH}$ 2.2.

It appeared, therefore, from the results of both the in vitro and the in vivo experiments, that the necessity for the use of such greater quantities of penicillin when administered orally could not be 


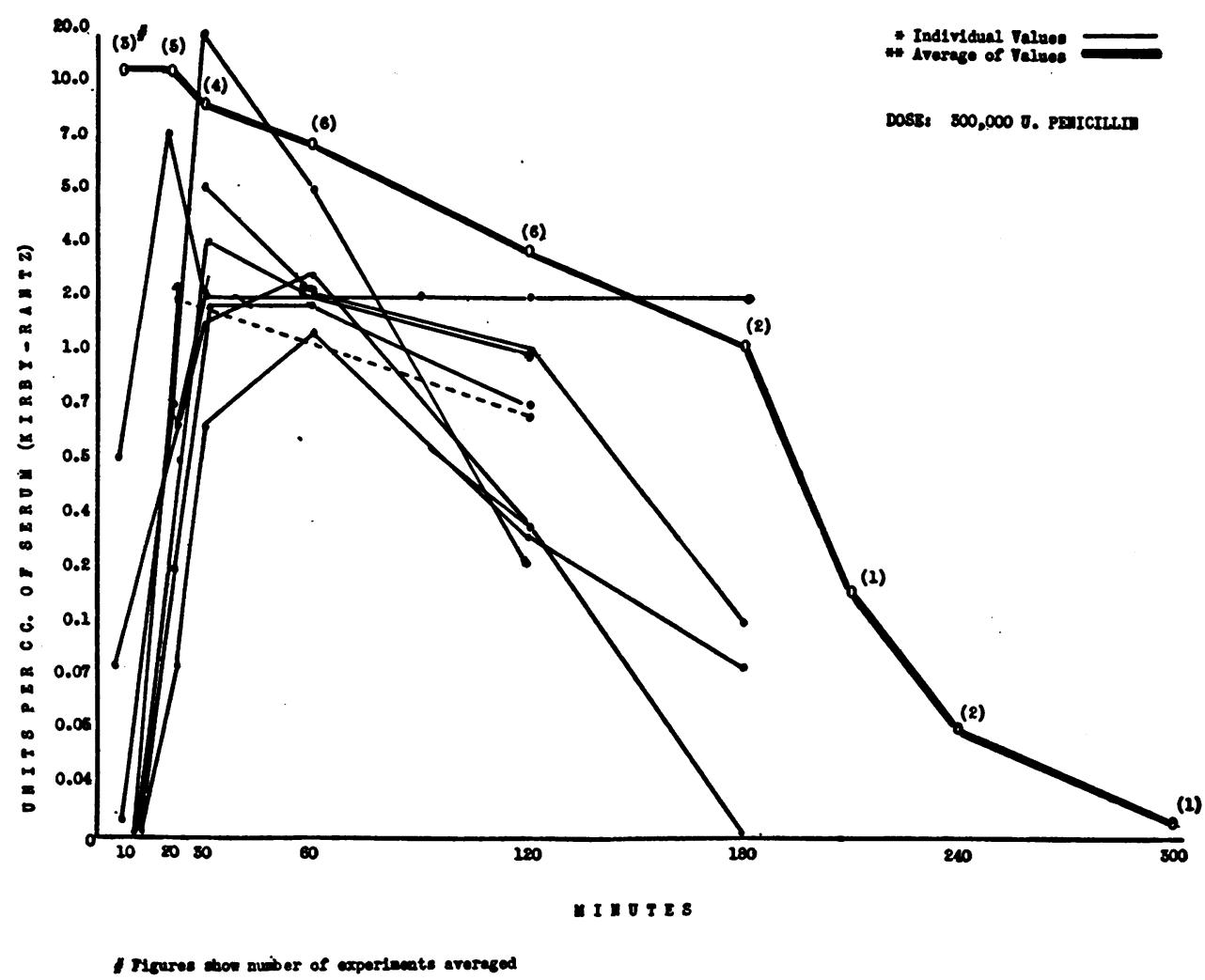

Fig. 5. Comparison of Penicillin Concentrations in Blood After Oral * and Intramuscular ** Administration to Subjects with Complete Achlorhydria

explained satisfactorily solely on the basis of inactivation by the acid of the stomach. Therefore, in order to investigate the problem further, a series of observations were made on individuals with complete achlorhydria.

\section{Observations on subjects with complete achlorhydria:}

Six subjects in whom there was no secretion of acid after a subcutaneous injection of $0.33 \mathrm{mgm}$. of histamine phosphate were studied. Five of the individuals had pernicious anemia. A preliminary report of the results of some of these observations has been presented elsewhere (16).

In each-subject a comparison was made of the amounts of penicillin which appeared in the blood and were excreted in the urine following the administration of the same dose of penicillin by both the oral and the intramuscular routes. Experiments were conducted in 2 subjects after both 25,000 unit and 300,000 unit doses. In the remaining 4 subjects, only the penicillin values attained after the 300,000 unit dose were studied. A total of 10 individual experiments were performed and the results may be seen in Figures 5 and 6.

\section{Penicillin concentrations in the blood:}

In Figure 5 are presented the average values of the penicillin concentrations which developed in the blood after intramuscular administration, and the individual values which were attained following oral administration. There was a considerable variation (extremes of 1.25 and 20.0 units per ml. of serum) in the maximum penicillin concentrations which developed after ingestion of the 300,000 unit dose. In 4 of the 8 experiments, the peak concentration ( 1.25 to 2.5 units per ml.) was no greater than has been observed following the ingestion of 300,000 units of penicillin by individuals with normal acid secretion. In the remaining 4 experiments ( 2 of which were in the same subject) the maximum penicillin concentrations attained after oral administration were definitely 


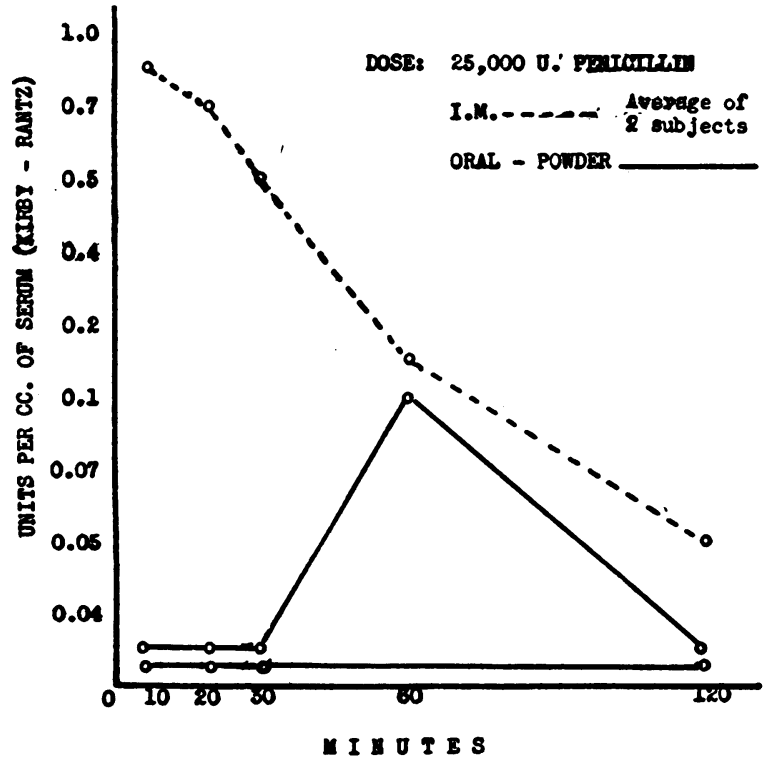

Fig. 6. Comparison of Blood Penicillin Concentrations Attained Following Oral and Intramuscular Administration of the Same Dose of Penicillin IN THE PRESENCE OF ACHLORHYDRIA

higher than has been observed following the ingestion of the same dose by normal individuals. In only 2 of these 4 experiments, however, were the peak concentrations attained after ingestion ( 7.0 and 20.0 units per ml. of serum) of the same order as occurred following intramuscular administration. Although some of the experiments were not continued for a period sufficient for an exact comparison, it appeared that the penicillin disappeared from the blood at approximately the same rate following both methods of administration.

The results of the 2 experiments at the 25,000 unit level of dosage are presented in Figure 6. In neither subject were the blood levels observed after ingestion of the penicillin comparable to those which appeared after intramuscular administration of the same dose of the material. It is of interest that in 1 of these 2 subjects no detectable ( 0.04 unit per ml.) concentration was present at any time during the experimental period. When this same subject ingested a 300,000 dose, the resulting concentrations of penicillin in the blood were within the range observed in normal individuals.

\section{Excretion of penicillin in the urine:}

In Figure 7 are presented the results of the studies of the urinary excretion of penicillin in these subjects with achlorhydria. As may be seen, the amount of penicillin excreted in the urine expressed as the percentage of the total dose ranged from 36 to 100 per cent following intramuscular administration, and usually was more than 60 per cent. Following ingestion, the range of the urinary excretion of penicillin varied between 10 and 32 per cent in specimens collected for 8 or more hours after administration. In the individual experiments, the difference between the amounts of penicillin which appeared in the urine after oral, and after intramuscular administration, is striking.

In Figure 8 are presented, for comparison, data on the urinary excretion of penicillin by a group of healthy individuals during a minimum period of 12 hours after ingestion of 300,000 units. The results are grouped according to the vehicle with which the penicillin was administered. The values presented in the Figure are representative of those obtained in a total of $\mathbf{3} 1$ individual experiments after penicillin doses ranging from 100,000 to 300,000 units. The extreme values which were determined after the use of each vehicle are included. As may be seen, regardless of the vehicle, the urinary excretion ranged from 3 to 32 per cent, and usually was between 10 to 20 per cent of the ingested dose.

Thus the range of the urinary excretion of orally administered penicillin was of the same order of magnitude in the achlorhydric individuals as in individuals with presumably normal acid secretion. This was true regardless of whether attempts were made to protect the penicillin against acid destruction.

\section{Urinary excretion as an approximate index of absorption:}

Martin and Kirby (17) have recently shown that, by the use of suitable dilutions in the technique of bioassay, it is possible to demonstrate the presence in the urine of 70 to 100 per cent of a single intramuscularly administered dose of penicillin and 80 to 100 per cent of a single intravenously injected dose. In this laboratory, with the use of a less precise technique than that employed by Martin and Kirby, it is usually possible to demonstrate the urinary excretion of 60 to 100 per cent of a single dose of penicillin during 
an 8 to 12 hour period immediately following intramuscular administration. It appears, therefore, that, under the conditions of virtually maximum absorption provided by parenteral administration, only a small proportion of a single dose of penicillin is destroyed in the body and the remainder is excreted in the urine. Thus the urin- ary excretion affords an approximate index of the amount of penicillin which is absorbed. There is no evidence that penicillin absorbed into the portal circulation is inactivated in the liver. Therefore, with due allowance for the lack of precision of the methods of bioassay, it appears that urinary excretion also affords an approximate index of

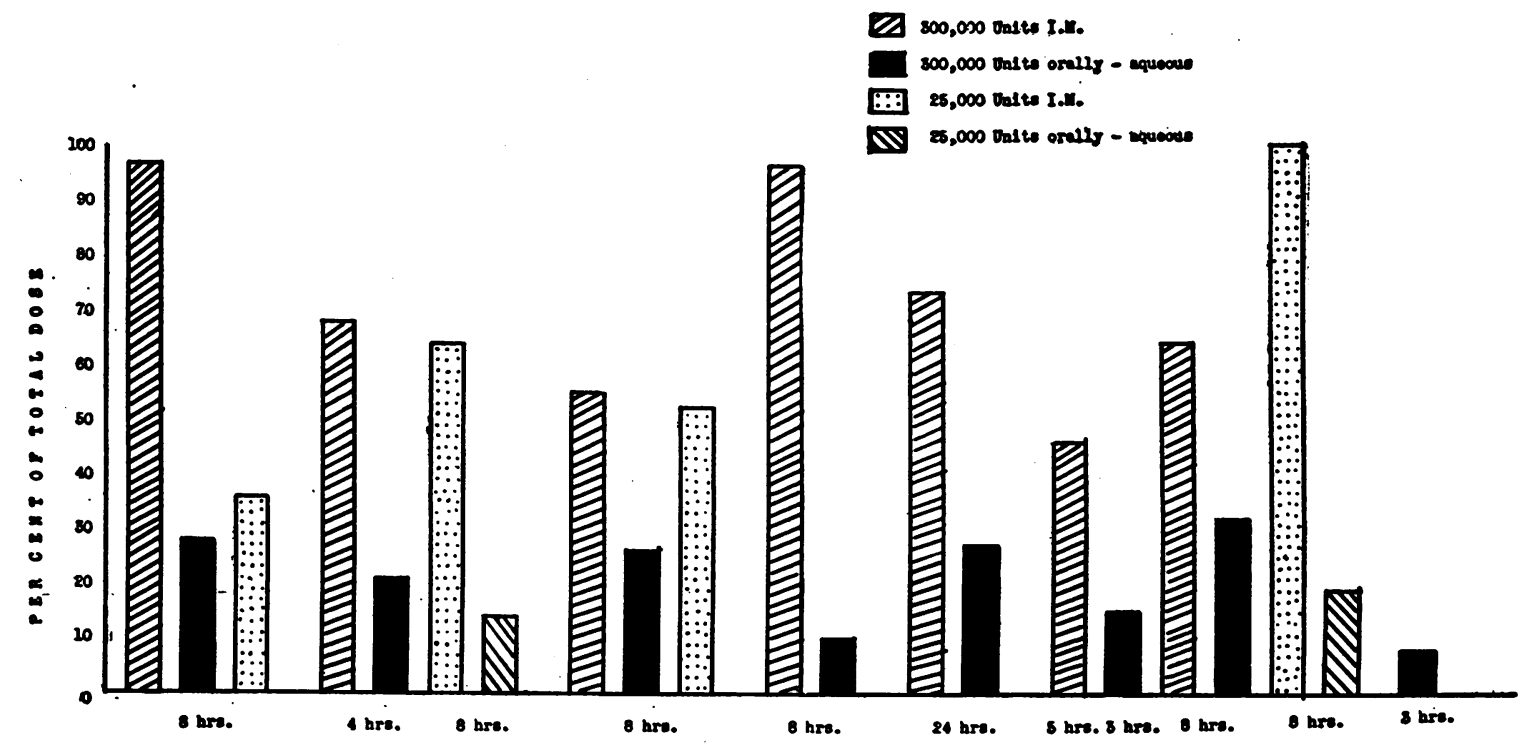

Fig. 7. Urinary Excretion of Orally Administered Penictllin in Individuals with Complete Achlorhydria

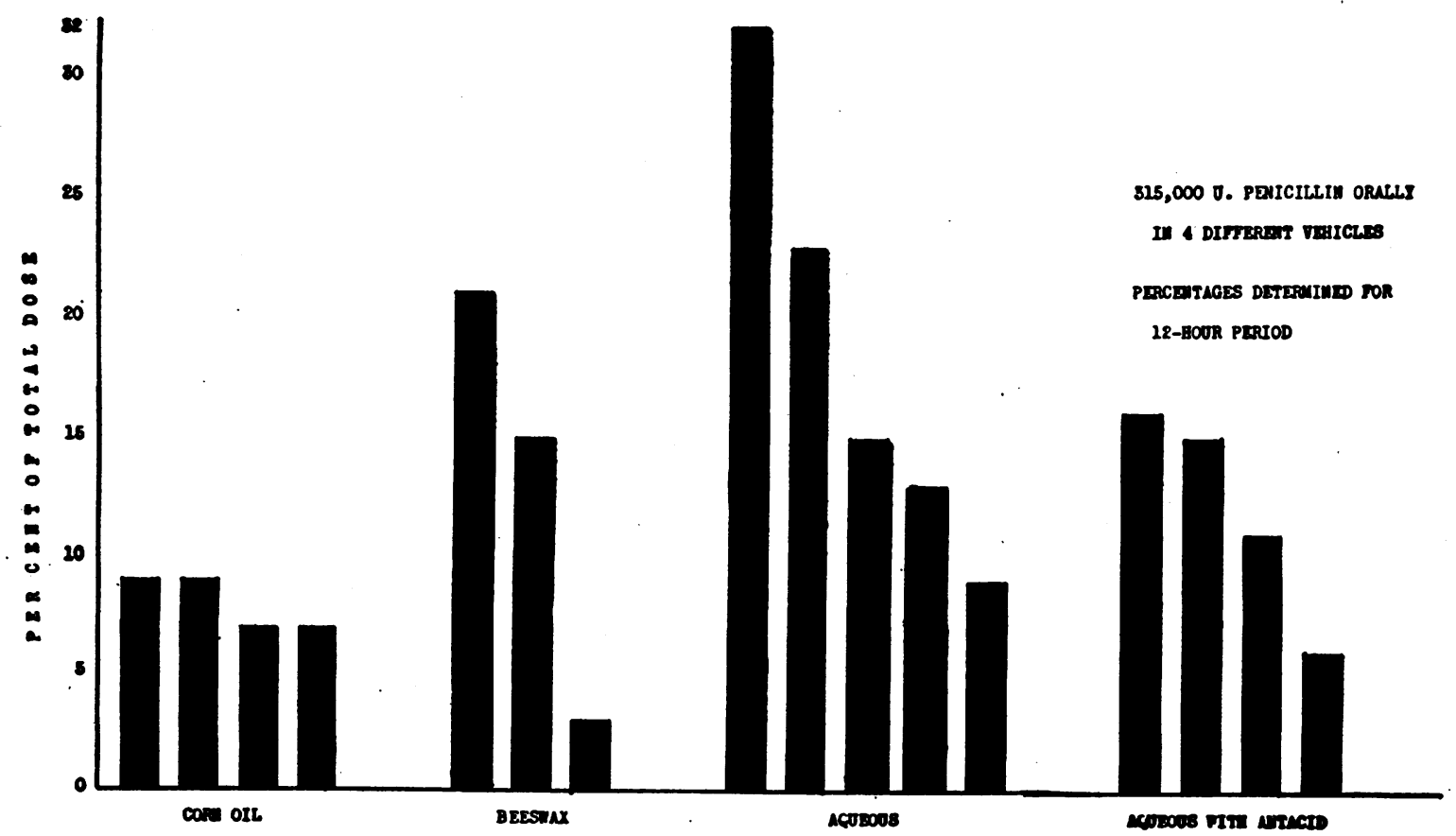

Fig. 8. Urinary Excretion of Orally Administered Penicillin in Normal Individuals 
absorption when penicillin is administered by mouth.

With the use of urinary excretion as an index of absorption, it is apparent from the preceding experiments that: $(a)$ the maximum absorption of an ingested dose of penicillin which has been observed represents only one-third of the total dose; (b) this maximum absorption which has been observed in individuals with complete achlorhydria has also been observed in individuals with presumably normal acid secretion whether or not acid-protective agents have been given; (c) that even in subjects with complete achlorhydria the portion of the ingested penicillin which is absorbed is usually appreciably less than the maximum absorption of approximately one-third; $(d)$ the twothirds or more of the orally administered penicillin which is not absorbed must either be destroyed within the body or excreted by a route other than through the kidney.

In the subjects with achlorhydria it was ojvious, and in the healthy individuals probable, that mechanisms other than destruction by the acid of the stomach must account for the disappearance of the majority of the ingested penicillin. Accordingly, an investigation of the absorption of the material from the intestine was made.

\section{Absorption of penicillin from the alimentary tract:}

Penicillin in aqueous solution, with or without an antacid, is absorbed rapidly from the alimentary tract of man following oral administration. Ten minutes after ingestion, appreciable concentrations of penicillin appear in the blood, and the maximum concentration is usually attained within 30 to 60 minutes (Figures 3 and 4). The peak concentration is seldom maintained for longer than 30 minutes. In man, a graph of the fall of the blood penicillin concentration usually assumes a straight line. It is not established how much or if any penicillin is absorbed from the stomach of man. Presumably in man, as in the cat, only a small amount of absorption would occur before the material has entered the duodenum. The latter event should take place within a short period (5 or 10 minutes) following ingestion by a fasting subject. Penicillin may be absorbed from any part of the small intestine of the cat, although the greatest absorption is from the duodenum.
In man, if orally administered penicillin is absorbed from the entire small intestine, it would be anticipated that: $(a)$ the slope of the fall of the blood penicillin concentrations from any given peak level would be less steep after oral than after parenteral administration; $(b)$ the urinary excretion of penicillin would remain high throughout the entire period that the material is present in the small intestine.

The following observations were made to test these hypotheses. On successive days individual subjects were given penicillin intramuscularly and orally. An oral dose of five times the intramuscular dose was used in an effort to obtain comparable concentrations of penicillin in the blood. In all but one of the experiments, large $(5,000,000$ unit) oral doses were employed in an effort to ensure the presence of an excess of penicillin in the intestine during the period of observation. During the first 8 or 12 hours of the experiments all urine voided in each 2 -hour period was collected as a separate specimen.

Rate of disappearance of penicillin from the blood:

In Figures 9, 10, and 11 may be seen the penicillin concentrations in the blood and the excretion in the urine after the administration of $5,000,000$ units orally and $1,000,000$ units intramuscularly. In all 3 experiments (which are representative), a penicillin concentration of 10.0 units per $\mathrm{ml}$. was present within the first hour after both methods of administration. In the succeeding 5 to 6 hours, the penicillin concentration fell rapidly from the 10.0 unit level to between 0.04 to 0.1 unit per $\mathrm{ml}$. Up to this point, the rate of the fall was identical, following both methods of administration. In 2 of the experiments, (Figures 9 and 10 ), the rate of disappearance was slowed after the fifth hour. As this phenomenon occurred after the administration of penicillin by both routes, its significance in regard to absorption from the intestine cannot be interpreted. There is evidence, however, that more penicillin was present in the intestinal tracts of these 2 subjects than in the subject of Figure 11. The latter became nauseated almost immediately after the ingestion of the $5,000,000$ unit dose of penicillin in aqueous solution, and vomited $5 \mathrm{~min}$ utes after the first specimen of blood was withdrawn, or 35 minutes after the administration 

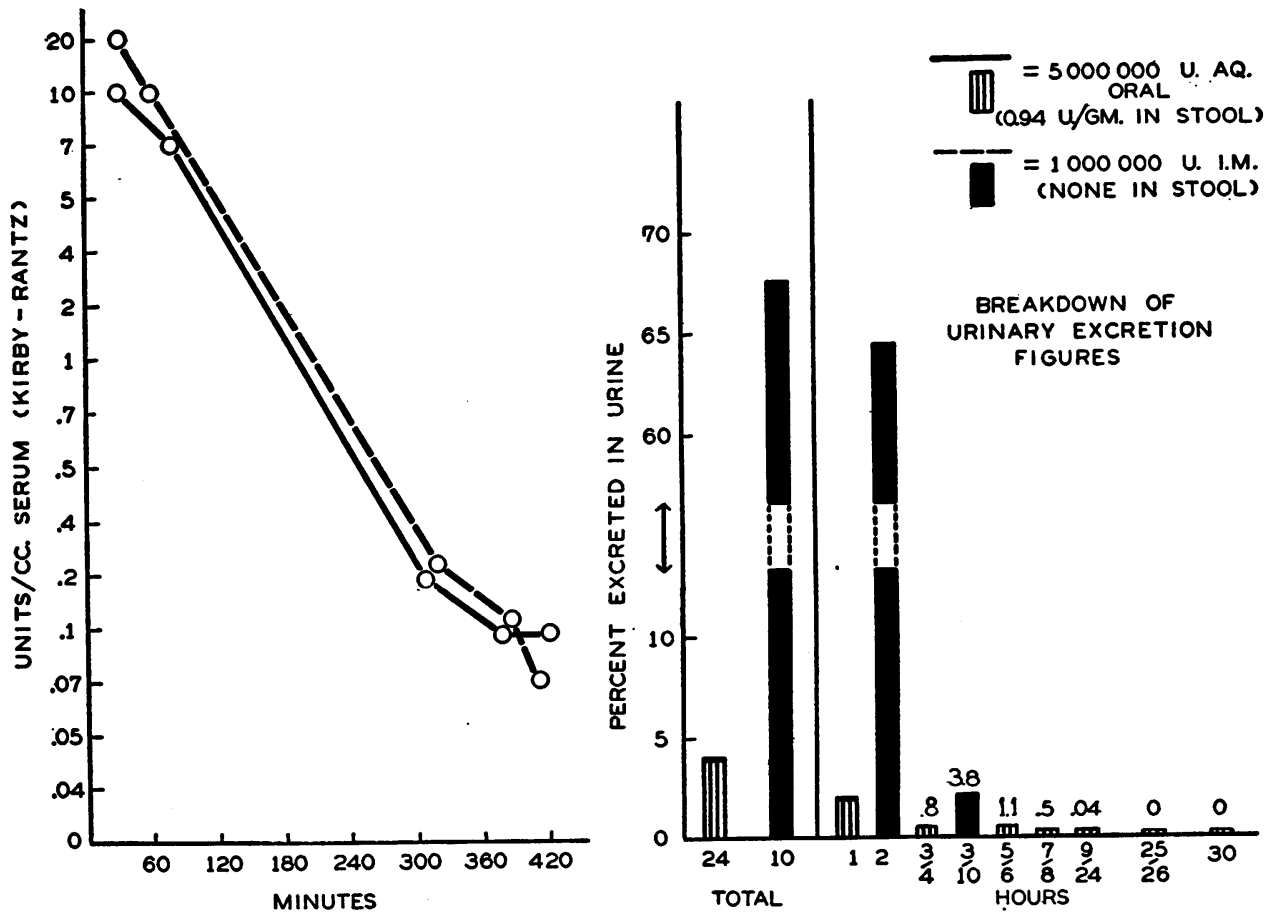

Fig. 9. Comparison of the Rate of Disappearance of Penicillin from the Blood and Its Appearance in the Urine Following Oral and Intramuscular Administration
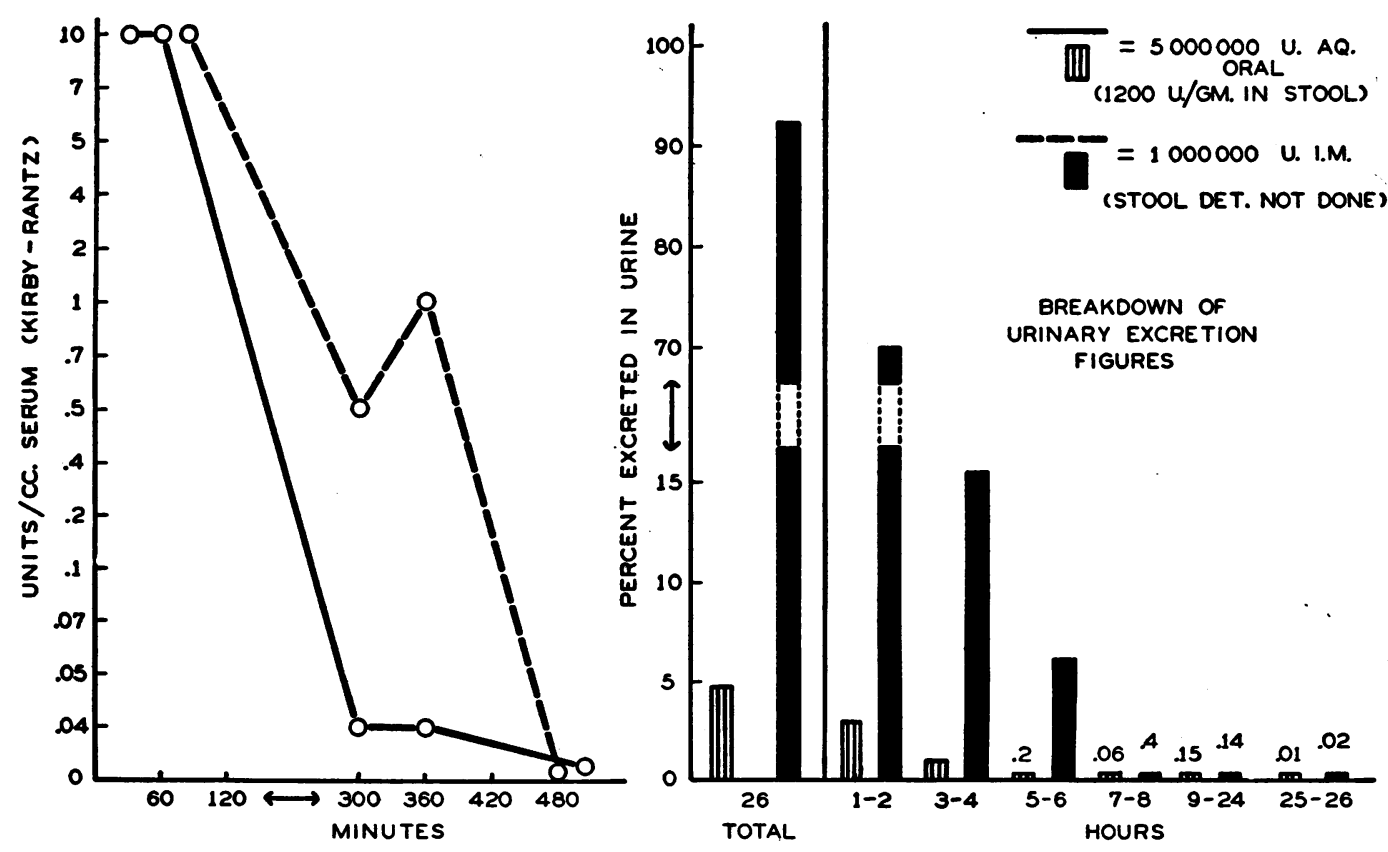

Fig. 10. Comparison of the Rate of Disappearance of Penicillin from the Blood and Its Appearance in the Urine Following Oral and Intramuscular Administration 

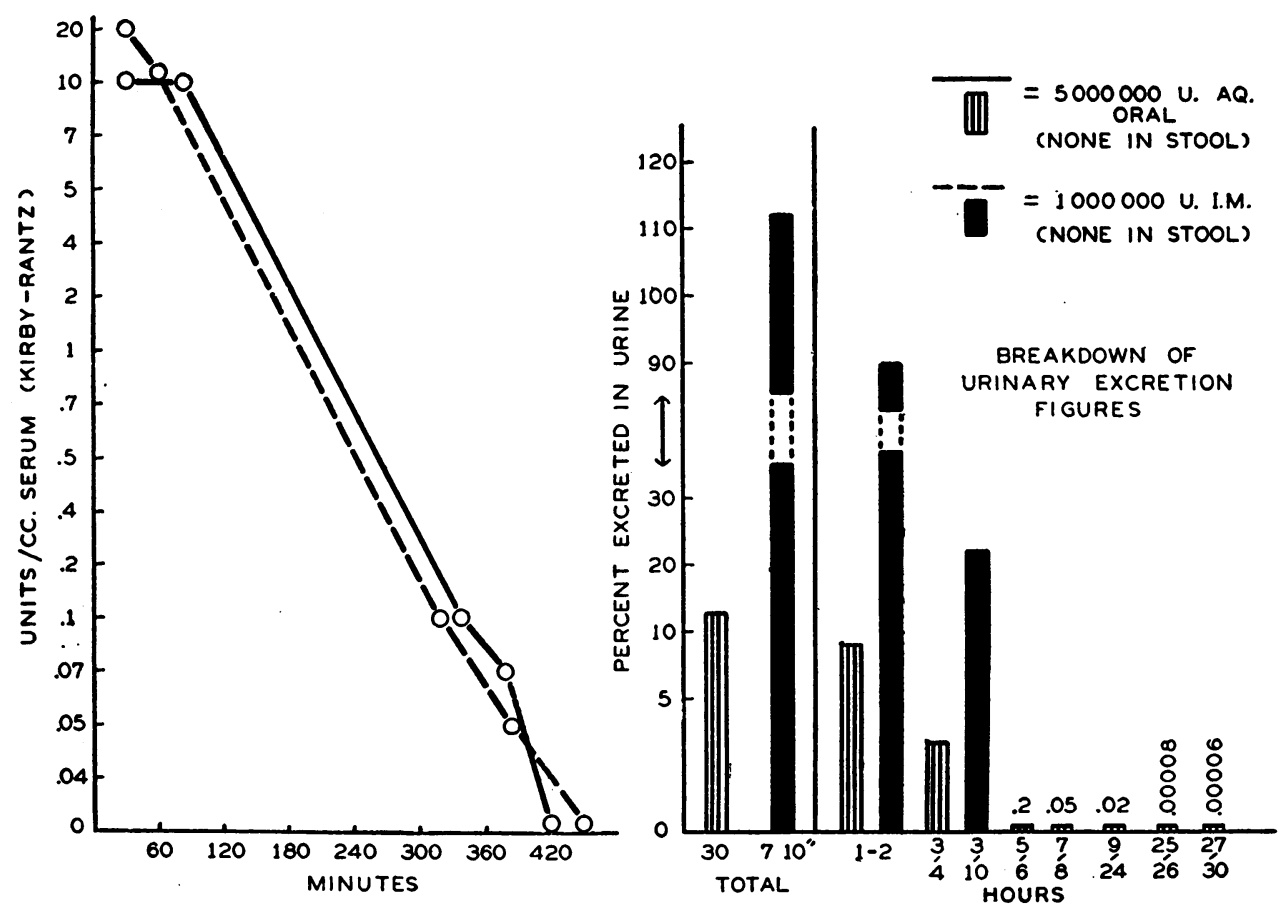

Fig. 11. Comparison of the Rate of Disappearace of Penicillin from the Blood and Its Appearance in the Urine Following Oral and Intramuscular Administration

of the penicillin. The volume and penicillin concentration of the vomitus were not measured, but the subject, who is a physician, estimated that he had lost "at least 90 per cent of the dose." Before emesis, a concentration of 10.0 units per ml. was present in the blood. Despite the fact that a large part, perhaps the majority, of the orally administered dose was not available for absorption, the fall in the penicillin concentration from the 10.0 unit level was at the same rate as from the 10.0 unit level attained after intramuscular administration in this subject. At six and a half hours after ingestion, a penicillin concentration of 0.07 unit per ml. was present. Furthermore, the slope of the curve of the blood penicillin levels attained in this subject who had lost a large part of the orally administered dose was virtually identical with those observed in the other subjects (Figures 9 and 10) who retained all of the ingested penicillin.

It appears that after the ingestion of penicillin the rate of fall of the concentration in the blood from a given level is virtually identical with the rate observed after intramuscular administration. This suggests that once the peak of absorption has been attained (within 30 or 60 minutes) subsequent absorption from the intestine is no greater than occurs during the same period from the site of an intramuscular injection.

\section{Excretion in urine:}

The amounts of penicillin which were excreted in the urine following both methods of administration are also presented in Figures 9, 10 and 11. As may be seen, after the intramuscular injection of $1,000,000$ units of penicillin, the urinary excretion in the 3 subjects was 68,93 , and 112 per cent respectively, as contrasted with an excretion of $4.0,4.5$, and 12.5 per cent respectively after ingestion of the 5,000,000 unit dose.

A comparison of the absolute amounts of penicillin excreted by these subjects after both routes of administration is of interest. The values for each subject respectively are : 225,000 units (oral) and 930,000 units (intramuscular) ; 200,000 units (oral) and 680,000 units (intramuscular) ; 600,000 units (oral) and 1,120,000 units (intramuscular). The discrepancy between the absolute amounts of penicillin excreted in the urine of each subject after the two methods of administration is 
probably a reflection of the crudity of the method of bioassay. There is a certain rough correlation, however, between these absolute values for urinary excretion, and the concentrations of penicillin which were demonstrable in the blood. In unpublished observations made in this laboratory, it has been noted that blood penicillin concentrations of essentially the same height and duration as were observed in the present experiments occur after the intramuscular administration of penicillin in doses ranging from 300,000 to 800,000 units. If it be assumed that each subject of the present experiments received as an intramuscular dose the same amount of penicillin as was excreted in the urine after either method of administration, the range of dosage $(200,000$ units to $1,120,000$ units) is not too far removed from the 300,000 to 800,000 unit range which produced blood penicillin concentrations of approximately the same order as were observed in the present experiments.

As may be seen in the figures, following either method of administration, almost all of the penicillin which appeared in the urine was excreted within the first 6 hours, at a time when detectable concentrations of penicillin were present in the blood. In the period from the seventh to the twenty-sixth hours inclusive, when the penicillin presumably had disappeared from the blood, minute amounts of penicillin continued to appear in the urine of the 3 subjects after oral administration. This continued excretion might represent the continued absorption from the lower intestine of amounts of penicillin too small to be detected in the blood. In the one subject in whom comparable data after intramuscular administration are available, however, similar minute amounts of penicillin continued to appear in the urine throughout the 26-hour period of observation. The continued urinary excretion of minute amounts of penicillin during the 24-hour period after an intramuscular injection of the material, is a phenomenon which has been observed frequently in investigations of the distribution of penicillin after intramuscular administration. It may represent a continued absorption from the site of the injection or the mobilization of small amounts of penicillin which were fixed in tissues. In the present experiments, it appeared that, once the initial excretion of the orally administered ma- terial was accomplished in the first 4 to 6 hours, subsequent excretion was so low as to indicate that little or no penicillin was entering the blood from the intestinal tract. It cannot be determined whether absorption occurred during the second to sixth hours inclusive. The penicillin excreted during that period may be merely a reflection of the high concentrations present in the blood during the first hour after ingestion. It would seem, however, from both the rate of disappearance of penicillin from the blood and its prompt excretion in the urine, that the pattern of absorption after oral administration is identical with that observed after intramuscular injection. This would indicate that most of the absorption occurs within a short period (approximately one hour) of ingestion. Thus the duration of the period in which penicillin remains in the blood after oral administration would be chiefly a reflection of the height of the concentration which was initially attained.

\section{Comparison of absorption after ingestion with ab- sorption from large intestine:}

In this experiment, on successive days, an identical amount of penicillin $(300,000$ units) was given: $(a)$ by mouth, and $(b)$ by direct introduction into the proximal end of the transverse colon through a colostomy. Observations of the absorption of the penicillin into the blood, and excretion in the urine were made as in the preceding experiments. The results are presented in Figure 12.

As may be seen in the Figure, following ingestion of the penicillin, absorption into the blood was rapid and a concentration of 2.0 units per $\mathrm{ml}$. was present at 30 and 60 minutes. The level fell gradually during the next 3 hours and the concentration was 0.04 unit per $\mathrm{ml}$. 4 hours after ingestion. When the same amount of penicillin was introduced directly into the large intestine, a concentration of 0.04 unit per ml. was attained 30 minutes after administration, but throughout the remainder of the 4-hour period of observation no penicillin could be detected in the blood. Additional evidence of the poor absorption from the large intestine is afforded by a comparison of the urinary excretion following the two routes of administration. After ingestion, 


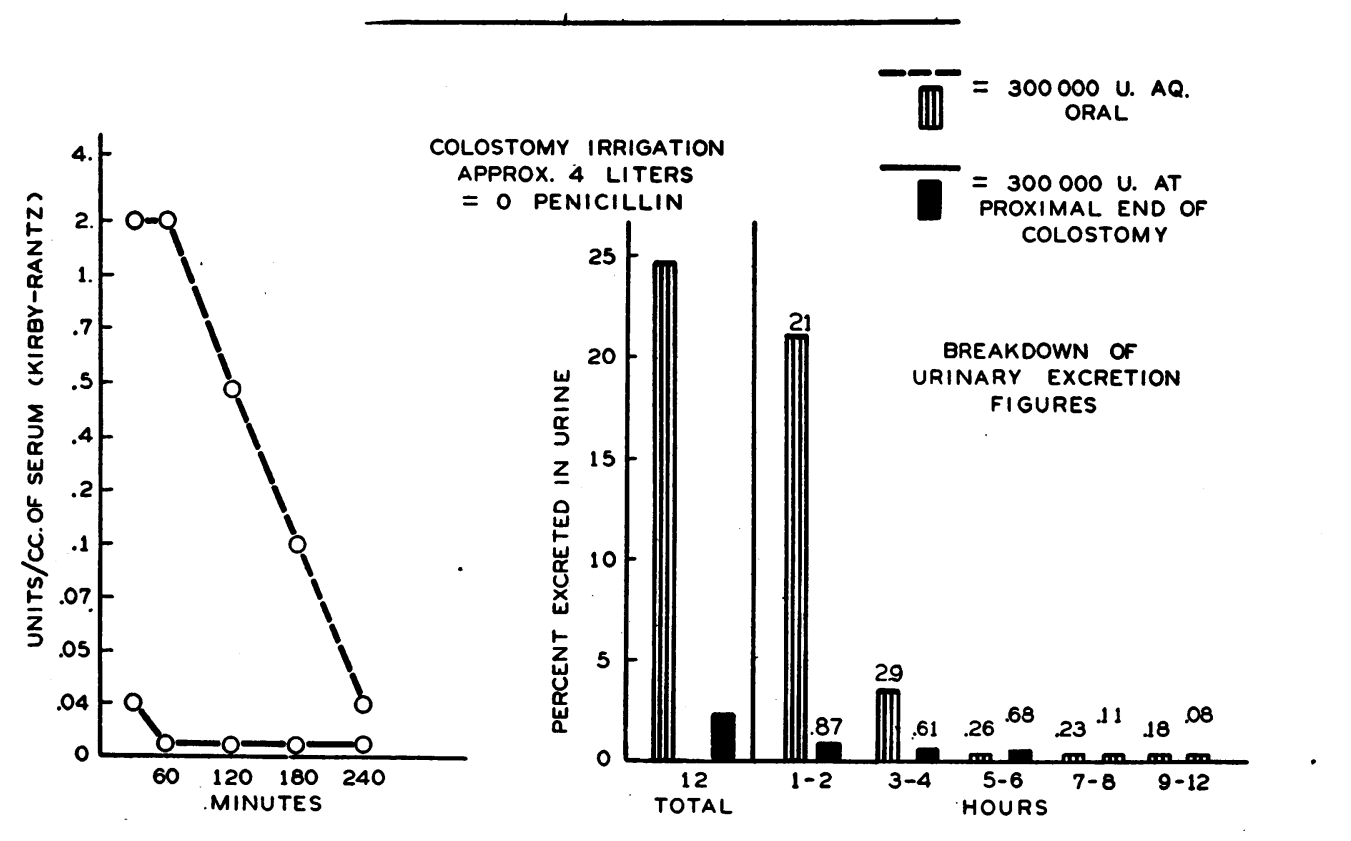

Fig. 12. Comparison of Absorption and Urinary Excretion of Penicillin Following: A. Ingestion; B. Introduction into the Large Intestine Through Colostomy

24.5 per cent of the total dose appeared in the urine during the succeeding 12 hours, in contrast to an excretion of 2.5 per cent in the same period when the penicillin was introduced directly into the large bowel. As in the experiments shown in Figures 9, 10, and 11, the greater part of the urinary excretion after oral administration occurred during the first 2 hours. A small but significant amount ( 3 per cent) was excreted during the third and fourth hours, and only minute amounts thereafter. In contrast, the urinary excretion after the penicillin was administered through the colostomy was maintained at the same low level ( 1 per cent) during the first 6 hours after administration, and only traces appeared thereafter.

The results of this experiment are qualitatively similar to those which were observed in cats (Figure 1). In addition, in the reports of several investigators $(3,18,19)$ who administered penicillin by rectum, only minimal concentrations of penicillin were attained in the blood in relation to the size of the doses which were administered. It appears, therefore, that in man as in the cat, absorption from the large intestine is poor.

\section{Destruction and excretion of penicillin in the intestine:}

As the majority of orally administered penicillin is not absorbed even in the absence of acid in the stomach, and as only insignificant absorption is possible once the 4 to 6 hour passage through the small intestine has been completed, it appeared that the penicillin which is neither destroyed by acid nor absorbed from the small intestine must be destroyed in the intestine or excreted in the feces.

The possibility of destruction in the upper part of the small intestine would seem to be unlikely because of the observations of Rammelkamp and Helm that penicillin is not inactivated in vitro by incubation with succus entericus or with bile.

Accordingly, the possibility of fecal excretion was investigated. Stool specimens which were obtained approximately 24 hours after administration of the penicillin in the experiments of the type presented in Figures 9, 10, and 11, were assayed for penicillin content. Determinations were made on stool specimens passed at the same interval after the intramuscular administration of penicillin to the same subjects. 


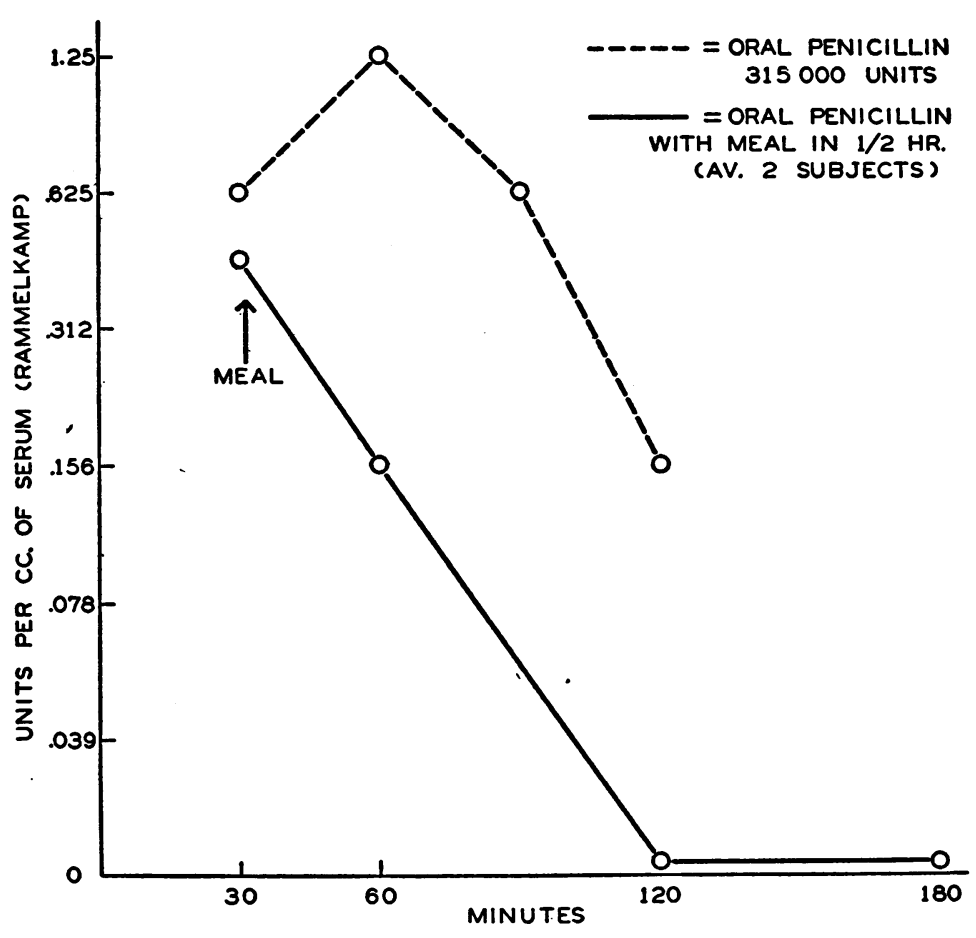

Fig. 13. Effect of Meal 30 Minutes After Ingestion of 300,000 Units of Penicillin

As may be seen in the figures, an appreciable amount of an antibacterial substance, presumably penicillin, was present in the stools of 2 of the 3 subjects after ingestion of the penicillin. Following the intramuscular administration of the material, no penicillin was detected in the stools from these subjects. The 1 subject (Figure 11) who failed to excrete penicillin in the stool after oral administration is the one who vomited a large part of the penicillin 35 minutes after ingestion.

There was a marked variation in the concentrations of penicillin which were present in the stools. In the 2 subjects who had received the 5,000,000 unit doses, the concentrations were 0.94 unit and 1,200 units per gram of stool, respectively. In contrast, the penicillin concentration in the stool of a subject who had ingested 300,000 units was 30.0 units per gram of stool. In another subject who had received 100,000 units of penicillin orally in aqueous solution at 2-hour intervals for a 24hour period before a specimen of stool was passed, the latter contained no penicillin.

Total stool collections for a period following the ingestion of the penicillin were not obtained in these experiments. It is evident, however, both from the marked variations in the concentration of penicillin excreted by this route, and from the height of the maximum concentration which was observed, that only a small fraction of the total dose can be accounted for on the basis of excretion in the feces.

In experiments which have been reported elsewhere (16), it was demonstrated that the incubation of penicillin with emulsions of stool results in the inactivation of 80 to 100 per cent of the penicillin within a 24 -hour period. The rate of inactivation varied but was usually considerable by the end of the third hour of incubation. The penicillin is presumably inactivated by bacterial action, as incubation of penicillin with Seitz filtrates of the stool emulsions caused little or no loss of penicillin activity.

\section{Effect of food upon absorption of penicillin:}

The absorption of orally administered penicillin is inhibited by the ingestion of food before the maximum penicillin concentration has been attained in the blood. In Figure 13 may be seen the blood penicillin concentrations attained after the ingestion of 300,000 units of penicillin by the 


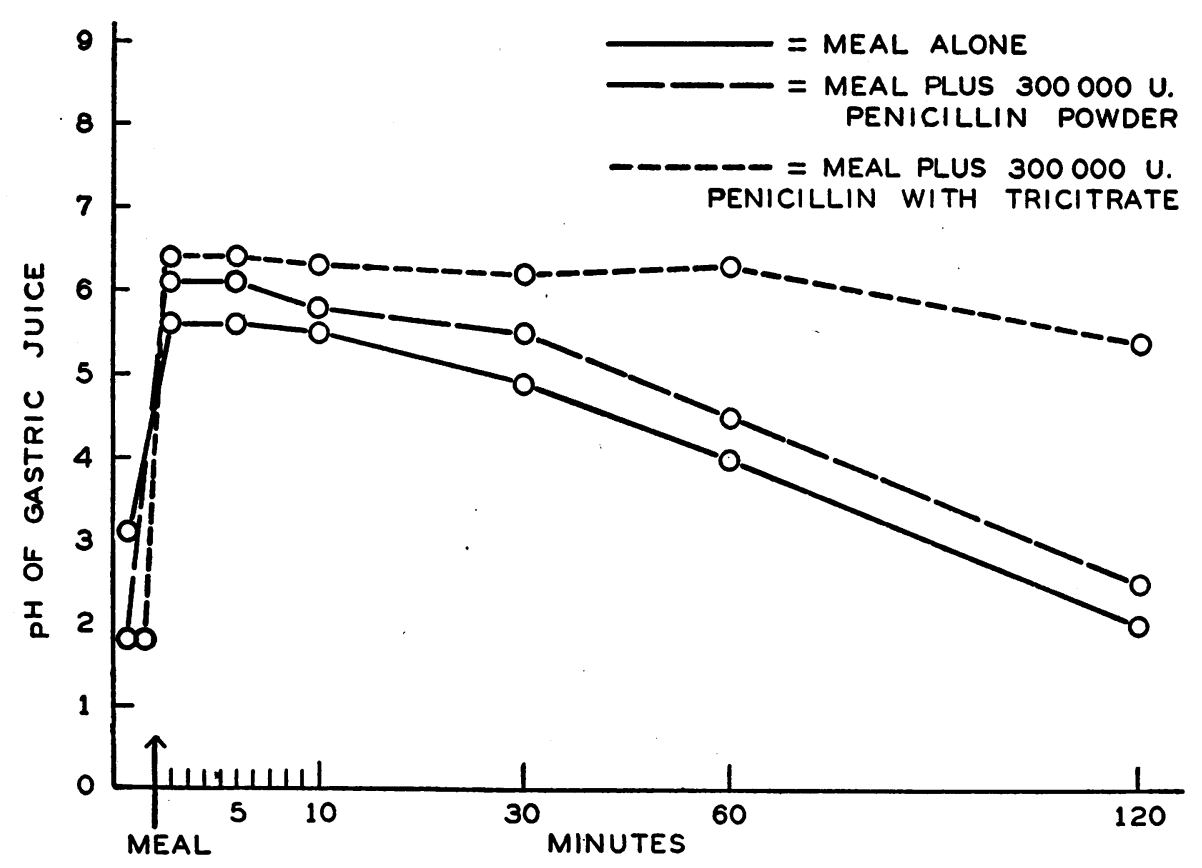

Fig. 14. Efrect of Food, Penicillin and Antacid on pH of Fasting Gastric Contents

same fasting subject: (a) when no food was ingested after administration of the penicillin; and (b) when a breakfast consisting of fruit juice, eggs, toast, and coffee was eaten 30 minutes after ingestion of penicillin.

The ingestion of food might conceivably inhibit the absorption of previously administered penicillin by increasing the gastric acidity, by decreasing gastric emptying, or by presenting a mechanical barrier to absorption from the stomach or small intestine. The first possibility (effect on $\mathrm{pH}$ ) is not likely, because of the known buffering action of food upon the gastric content. In Figure 14 may be seen the effect upon the $\mathrm{pH}$ of the gastric content of: (a) a standard mixed meal alone; (b) the standard meal followed immediately by 300,000 units of penicillin in water; (c) the standard meal followed immediately by 300,000 units of penicillin sodium tricitrate tablets. The experiments were performed in the subject with a permanent gastric fistula, on successive days, under standard conditions. As may be seen in the figure, the fasting levels of $\mathrm{pH}$ of $1.9,1.9$, and 3.0 were promptly raised to a $\mathrm{pH}$ of 5.5 or higher immediately after ingestion of the food whether or not penicillin or penicillin-tricitrate was also administered.
The $\mathrm{pH}$ persisted at the higher level for a longer period in the experiment in which the antacid was used. In all 3 experiments, however, the $\mathrm{pH}$ of the gastric content remained above 4.0 for a period of 60 minutes after the meal.

The second possibility (decrease in gastric emptying) cannot be excluded. It is probable, however, that the larger part of an orally administered dose of penicillin usually enters the duodenum within the first 30 minutes after ingestion by a fasting subject.

It appears, therefore, that the third possibility (mechanical interference in the stomach or small intestine) is the most probable explanation for inhibition of absorption produced by the ingestion of food 30 minutes after the oral administration of penicillin.

\section{DISCUSSION}

On the basis of the experimental results which have been presented, it seems permissible to attempt to reconstruct the sequence of events which follow the oral administration of penicillin.

Immediately after ingestion, the material passes into the stomach. The extent of penicillin absorption which occurs from the stomach of man has not been established. In the isolated stomach of the cat, absorption occurs but is not so great as from 
the small intestine. Aside from the problem of gastric absorption, a variable amount of the penicillin which enters the stomach may be inactivated. The quantity of penicillin which is destroyed by the acid of the stomach probably varies within a wide range and is conditioned by several factors. Important among these are: the $\mathrm{pH}$ of the gastric content, and the time during which the penicillin is subject to the possible effects of that particular hydrogen ion concentration, i.e., the speed of gastric emptying.

It has been observed that the destruction of penicillin in gastric juice in vitro, is rapid at $\mathrm{pH}$ 2.0 , requires several hours for completion at $\mathrm{pH}$ 2.0 to 4.0 , and proceeds only slowly at $\mathrm{pH} 4.0$ or above. It has also been observed in human subjects that the $\mathrm{pH}$ of the gastric content fluctuates rapidly and is frequently at 4.0 or above. Information on the usual length of stay of penicillin in the stomach is less clear cut, but apparently the greater part of a dose ingested into an empty stomach enters the duodenum within an hour. Because of these several variables, it has not been possible to establish the exact amount of penicillin which is actually destroyed by the acid of the stomach, but apparently the degree of destruction is seldom great.

Absorption of penicillin into the blood first becomes evident within 10 to 20 minutes after ingestion, and the maximum blood concentration develops within the first hour. Once this peak concentration is reached, it persists for only a short period, usually less than 30 minutes. The subsequent fall in the level of the penicillin in the blood is rapid, and occurs at the same rate whether the peak level was produced by oral or by intramuscular administration. This suggests that significant amounts of penicillin are not absorbed into the blood after the peak concentration has been attained and that in man, as in the cat, the greatest amount of absorption occurs from the duodenum.

Whether penicillin is absorbed from the jejunum or ileum has not been established. In the cat, when the penicillin was confined to these areas, the resulting absorption was almost as extensive as occurred from the duodenum. Even when the material is continually progressing through the small intestine, as occurs in man, it would be anticipated that there would be an adequate period of time for absorption from these areas (ileum and jejunum). Yet from the studies of the pattern of absorption and urinary excretion of ingested penicillin in man, there is little to indicate that such continued absorption occurs. Even in the subjects with achlorhydria, the rate of disappearance of penicillin from the blood was apparently the same after oral and intramuscular administration. Penicillin is available for absorption throughout the small intestine, as a large part of ingested material is not absorbed at all. This is established by the demonstration that penicillin may be excreted in the feces.

The possibility that penicillin is absorbed from the small intestine but is destroyed in the liver would seem to be excluded by the concentrations which developed in the systemic circulation following the direct injection of penicillin into the portal vein.

Regardless of whether penicillin is absorbed from the small intestine distal to the duodenum, only insignificant amounts are absorbed once the material enters the large bowel. This fact is evident from the studies of absorption after the introduction of penicillin into the large intestine of the subject with a colostomy, and the observations in cats. Confirmatory evidence is supplied by the fact that in the subjects who ingested large doses of penicillin, only minute amounts were excreted in the urine subsequent to the sixth hour after ingestion, although appreciable quantities of penicillin were subsequently passed in the feces.

After penicillin has entered the large bowel, subsequent absorption is negligible, and the material is exposed to the destructive action of the feces. This phenomenon, which was originally noted by Abraham et al. (1), has been described in a previous publication (16). Although in vitro the rate of inactivation of penicillin by an emulsion of stool is variable, it is usually considerable within 4 or 5 hours and attains 80 to 100 per cent effectiveness within 24 hours. Penicillin which survives the destructive action of the lower intestinal contents is excreted in the feces.

It is not possible to establish with precision the relative amounts of an orally administered dose of penicillin which are destroyed or absorbed at the various sites which have been described. The maximum absorption (as judged by urinary excretion) observed in the patients with complete achlorhydria was only one-third of the ingested 
dose. In those subjects the entire ingested dose presumably entered the small intestine. If it can be assumed that the absorption of penicillin from the small intestine of individuals with normal acid secretion (neutralized or unneutralized) proceeds in the same manner as in the subjects with achlorhydria, the maximum absorption under any conditions would represent approximately onethird of the amount of penicillin which has entered the duodenum.

Obviously, the proportion of the ingested dose which enters the duodenum depends upon the extent of destruction which may have occurred in the stomach. These two variables (respective quantities absorbed, or inactivated by acid) cannot be separated for analysis on the basis of the available evidence. It is possible only to compare the amounts of penicillin which are absorbed under varying conditions, and from those data to make inferences as to the amount which may have been destroyed prior to absorption. In the following proposition this method of calculation is used.

The blood penicillin concentrations and the quantitative urinary excretion are of the same order of magnitude in fasting subjects after ingestion of penicillin with or without acid neutralizing agents. Similar determinations in achlorhydric subjects reveal strikingly higher values for the concentrations of penicillin in the blood in a few instances, but "normal" or only slightly higher values in the majority of the experiments. In these achlorhydric subjects the values for the urinary excretion of penicillin are no greater than the maximum observed in subjects with normal acid secretion. As the amounts of penicillin which are absorbed under these varying conditions are of the same general order of magnitude (save for the exceptions noted), it appears that the amount of penicillin which is usually destroyed in the stomach represents only a small fraction of the ingested dose (probably in the neighborhood of 10 or 15 per cent).

It would follow from this that the use of acid protective agents in the oral administration of penicillin constitutes an attack upon only a small portion of a problem which is chiefly one of absorption. The theoretical maximum advantage which could be attained from the use of such agents could be only to promote a consistent ab- sorption of penicillin of the same order as the maximal absorption which has been noted in the complete absence of gastric acid, i.e., 32 per cent urinary excretion. Unless a protective agent could promote a consistent absorption (urinary excretion) of approximately 30 per cent (which has not been observed as yet), it would have only limited value.

An estimation of the proportion of the ingested dose which is eventually inactivated in the large intestine or excreted in the stool can also be made only by inference. The maximum absorption (i.e., urinary excretion) which has been observed under any conditions ( 32 per cent) represents only one-third of the ingested dose. In the achlorhydrics, at least two-thirds, and in the other individuals almost as large a portion of the total ingested dose must be destroyed in the colon or excreted in the feces.

As it appears that a failure in maximum absorption from the small intestine is the most important factor in the relative inefficiency of the oral route for the administration of penicillin, it would seem that attempts to either (a) prolong the stay of penicillin in the upper intestine, or $(b)$ allow the slow release of penicillin during its passage through the small intestine, might result in a more efficient preparation for oral use. Thus far, the use of agents such as beeswax, shellac, or stearates for these purposes has proven disappointing. Beeswax was originally used by Romansky and Rittman (20) to retard the absorption of penicillin from an intramuscular injection. At times the oral administration of oil suspensions of penicillin mixed with either beeswax or shellac (or both) will result in penicillin concentrations in the blood which persist for significantly longer periods than those which have been observed after any other method of oral administration. Usually, however, although absorption may be delayed, it is no greater than occurs after the ingestion of penicillin alone. Occasionally absorption is retarded sufficiently so that detectable concentrations do not appear in the blood, although small amounts are excreted in the urine. From the observations of the present investigation, it would seem that a possible source of failure in the use of absorption "retarders" for oral administration might be that the penicillin is held in combination until the vehicle leaves the duodenum. If the penicillin- 
"retarder" combination were such that penicillin were not released for several hours, the mixture might be carried into the cecum, from which only minimal absorption occurs.

Further investigation of the use of these agents such as beeswax for the slow release of penicillin would seem to be a rational approach to the problem of increasing the efficiency of the oral route. However, until it can be established that absorption from such a vehicle is consistently regular, it should not be used for the treatment of serious infections.

As the purpose of the present investigation has been to study the ultimate fate of orally administered penicillin, emphasis has been laid on the variability in the extent of destruction and absorption, and on the limitations of the amount of absorption, which occur following the ingestion of penicillin as the powder or in the various combinations studied. It should not be inferred from this that oral administration is not a practicable method for the administration of penicillin. Variability of absorption is a phenomenon common to many agents which are regularly administered by mouth, and occurs, within limits, following the intramuscular administration of penicillin. The absorption of orally administered penicillin also varies within limits, but is reasonably consistent and predictable, provided that sufficiently large doses are used. The chief danger at present in the oral administration of penicillin is that, in an effort to demonstrate that a particular vehicle "protects" the penicillin from destruction, dangerously inadequate penicillin regimens will be advocated. Until it can be demonstrated that more than a third of the penicillin is absorbed from a single dose of a given preparation, it will be necessary to adhere to the oral-intramuscular ratios based on the studies of absorption. As presented above, the range of this oral-intramuscular ratio is approximately from 10 to 1 , to 3 to 1 . (A 5 to 1 ratio has been satisfactory in clinical practice.) If sufficient material is administered, however, it is possible, with no serious discomfort to the patient, to attain, by oral administration, concentrations of penicillin in the blood of the same height and duration as are produced following intramuscular administration.

\section{SUMMARY AND CONCLUSIONS}

An attempt has been made to trace the fate of orally administered penicillin in an effort to establish the reason for the relative inefficiency of this route for the administration of penicillin. On the basis of the observations which have been presented, it is concluded that:

1. Absorption of ingested penicillin occurs chiefly from the duodenum. The amount of absorption which occurs from the stomach is not established, but is probably small.

2. Inactivation of penicillin as a result of the acidity of the gastric content is conditioned by a number of variables, and on the whole is seldom great.

3. Absorption of penicillin is rapid. The maximum concentrations are attained in the blood within thirty to sixty minutes of ingestion. The subsequent persistence of penicillin in the blood is a reflection of the height of the maximum concentration originally attained, and does not appear to be a result of continued absorption from the alimentary tract.

4. Absorption of ingested penicillin is incomplete. Two-thirds or more of an orally administered dose are apparently not absorbed.

5. Once penicillin has passed through the small intestine, only insignificant amounts are absorbed.

6. The penicillin in the intestine which is not absorbed is inactivated by the bacteria in the colon, or, if an excess be present, it is excreted in the feces.

7. The necessity for the use of larger amounts of penicillin by the oral than by the intramuscular route is primarily the result of incomplete absorption and cannot be explained satisfactorily on the basis of destruction by acid or bacterial action.

The authors wish to express their thanks to Miss Helen Steinberg for her valuable assistance in this investigation.

\section{BIBLIOGRAPHY}

1. Abraham, E. P., Chain, E., Fletcher, C. M., Gardner, A. D., Heatley, N. G., Jennings, M. A., and Florey, H. W., Further observations on penicillin: Growth, assay, production, bacteriostatic action, effect on cells, absorption and excretion and therapeutic trial. Lancet, 1941, 2, 177.

2. Florey, M. E., and Florey, H. W., General and local administration of penicillin. Lancet, 1943, 1, 387. 
3. Rammelkamp, C. H., and Keefer, C. S., Absorption, excretion and distribution of penicillin. J. Clin. Invest., 1943, 22, 425.

4. Rammelkamp, C. H., and Helm, J. D., Jr., Studies on the absorption of penicillin from the stomach. Proc. Soc. Exper. Biol. and Med., 1943, 54, 324.

5. Powell, H. M., and Jamieson, W. A., Response of sulfonamide-fast pneumococci to penicillin. Proc. Soc. Exper. Biol. and Med., 1942, 49, 387.

6. Libby, R. L., Oral administration of penicillin in oil. Science, 1945, 101, 178.

7. Free, A. H., Leonards, J. R., McCullagh, D. R., and Biro, B. E., The urinary excretion of penicillin after oral administration to normal human subjects. Science, 1944, 100, 431.

8. Little, C. J. H., and Lumb, G., Penicillin by mouth. Lancet, 1945, 1, 203.

9. Charney, J., Alburn, H. E., and Bernhart, F. W., Urinary excretion of penicillin in man after oral administration with gastric antacids. Science, 1945, 101, 251.

10. György, P., Vandegrift, H. N., Elias, W., Colio, L. G., Barry, F., and Pilcher, J. D., Administration of penicillin by mouth. Preliminary report. J. A. M. A., 1945, 127, 639.

11. McDermott, W., Bunn, P. A., Benoit, M., DuBois,
R., and Haynes, W., Oral penicillin. Science, 1945, 101, 228.

12. Rammelkamp, C. H., A method for determining the concentration of penicillin in body fluids and exudates. Proc. Soc. Exper. Biol. and Med., 1942, 51, 95.

13. Kirby, W. M. M., and Rantz, L. A., Methods of measuring penicillin concentrations in body fluids. J. Bact., 1944, 48, 603.

15. Wolf, S., The relation of gastric function to nausea in man. J. Clin. Invest., 1943, 22, 877.

16. McDermott, W., Bunn, P. A., Benoit, M., DuBois, R., and Reynolds, M. E., The absorption of orally administered penicillin. Science, In press.

17. Martin, S. P., and Kirby, W. M. M., Urinary excretion of penicillin following single parenteral injections. To be published.

18. Fleming, A., Micro methods of estimating penicillin in blood serum and other body fluids. Lancet, 1944, 2, 620.

19. Loewe, L., Alture-Werber, E., and Rosenblatt, P., Administration of penicillin by rectal suppository. Preliminary note. J. A. M. A., 1945, 128, 18.

20. Romansky, M. J., and Rittman, G. E., A method of prolonging the action of penicillin. Science, 1944, 100, 196. 